\title{
Demanda en servicios pediátricos de un hospital del Callao, Perú
}

\section{Demand for pediatric services in a hospital in Callao, Peru}

Correspondencia Manuel André Virú Loza m.andre.viru@gmail.com

Recibido: $27 / 10 / 2018$ Aprobado: 07/11/2018

Citar como: Virú-Loza MA. Demanda en servicios pediátricos de un hospital del Callao, Perú. Acta Med Peru. 2018;35(4):244-5

\author{
Manuel A. Virú-Loza ${ }^{1 a}$ \\ 1 Universidad Nacional Mayor de San Marcos. Lima, Perú. \\ a Médico pediatra
}

\section{Sr. Editor:}

Existen estudios sobre la sobredemanda de los servicios de emergencia en el Perú ${ }^{[1,2]}$; sin embargo, hasta donde se tiene conocimiento, es escasa la información disponible respecto a la demanda de servicios pediátricos.

Con el fin de aportar información al problema planteado se analizaron datos provenientes del Hospital Nacional Daniel Alcides Carrión del Callao disponibles en su página web institucional ${ }^{[3]}$. Se muestra cómo ha ido evolucionando el número de atenciones en la emergencia pediátrica, en los consultorios de pediatría y el número de recién nacidos vivos a lo largo de los años. Se presenta información de los meses y años para los cuales estaba disponible la información de estos tres indicadores simultáneamente (Figura 1).

El número de atenciones en la emergencia pediátrica tuvo una tendencia al incremento en los últimos años. Comparando el periodo enero a junio de 2011 con el correspondiente al 2018, hubo un incremento de 6889 atenciones, lo que representó, en promedio, 38 atenciones más al día. Esto podría conllevar a una sobresaturación de este servicio en caso no se hubiesen tomado las medidas del caso en el mismo periodo

En relación al número de nacimientos, el hecho de no observar una tendencia hacia incremento o disminución no necesariamente implica que en la región los nacimientos se hayan mantenido de igual forma, a pesar de que se trata de un hospital de referencia. Por lo tanto, dicha información es insuficiente para plantear la posibilidad de alguna relación entre el número de atenciones en la emergencia pediátrica y el número de nacimientos en la región en los últimos años. Lo que sí resalto es que en el servicio de neonatología, no parece haber una tendencia que sugiera un aumento significativo de la demanda de recién nacidos vivos tomando como referencia a los años previos.

Finalmente, el número de atenciones por consultorio externo tuvieron una disminución notoria entre los años 2012 y 2017. Comparando el periodo enero a junio de 2012 con el correspondiente al 2017, hubo una disminución de 3508 atenciones, lo que representó, en promedio, 19 atenciones menos al día.

En el extranjero, se vio que el uso recurrente de la emergencia pediátrica era más común en aquellos niños con seguro de salud público y con problemas de salud no tan agudos ${ }^{[4]}$, así como aquellos cuyo apoderado tenia un bajo nivel de conocimiento en temas de salud ${ }^{[5]}$.

En la práctica clínica, algunas veces los pacientes son atendidos en el servicio de emergencia por problemas que normalmente podrían resolverse en un consultorio externo. En este hospital, optimizar 


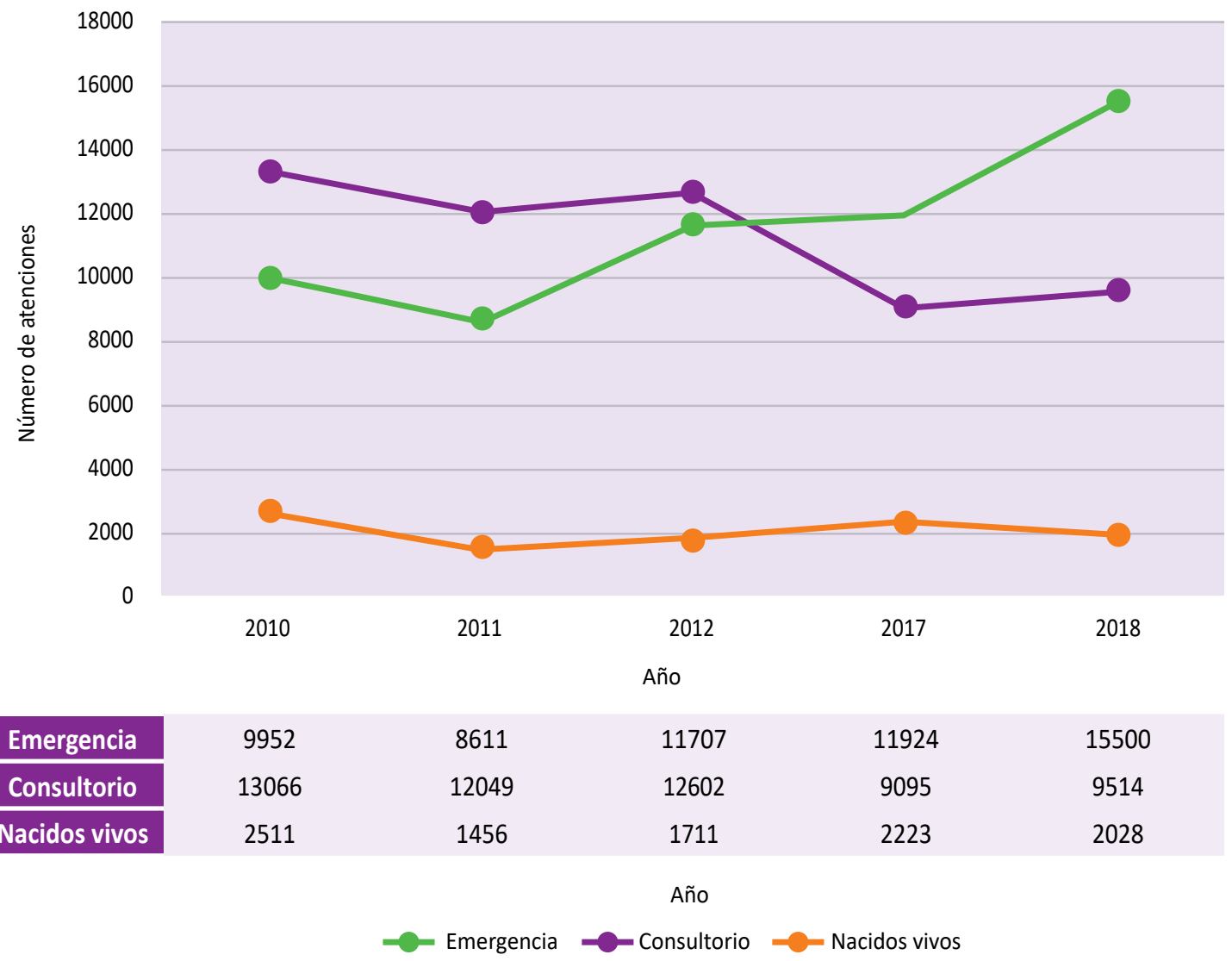

* Para cada año se consideraron las atenciones brindadas entre los meses de enero a junio.

Figura 1. Número de atenciones por emergencia pediátrica, número de atenciones por consultorio de pediatría y número de nacidos vivos en los periodos enero a junio de los años 2010, 2011, 2012, 2017 y 2018.

la atención por consultorio podría reducir al menos parcialmente el número de atenciones por emergencia, reduciendo el riesgo de sobresaturación e incrementando la eficiencia de la misma.

Es probable que en otros centros se haya dado escenarios diferentes con respecto a la variación de atenciones en estos tres servicios pediátricos. Por lo tanto, es importante detectar tendencias y estudiarlas teniendo en cuenta las variaciones que pueden existir en diferentes poblaciones y así formular políticas sanitarias que se adapten a diversos contextos.

Financiamiento: Autofinanciado.

Conflicto de intereses: El autor refiere haber laborado en el servicio de pediatría del Hospital Nacional Daniel Alcides Carrión entre julio de 2015 y junio de 2018, por motivo de residencia médica.

\section{REFERENCIAS BIBLIOGRÁFICAS}

1. Ponce-Varillas TI. Hacinamiento en los servicios de emergencia. An Fac med. 2017;78(2):218-23.

2. Vásquez-Alva R. Sobredemanda de atención médica en el servicio de emergencia de adultos de un hospital terciario, Lima, Perú. An Fac med. 2016;77(4):379-85.

3. Hospital Nacional Daniel Alcides Carrión. Sala Situacional Virtual [Internet]. Bellavista: Hospital Nacional Daniel Alcides Carrión; c2018 [citado el 10 de octubre de 2018]. Disponible en: http:// www.hndac.gob.pe/web/sala-situacional-virtual.html

4. Neuman MI, Alpern ER, Hall M, Kharbanda AB, Shah SS, Freedman $S B$, et al. Characteristics of recurrent utilization in pediatric emergency departments. Pediatrics. 2014;134(4):e1025-31.

5. Morrison AK, Schapira MM, Gorelick MH, Hoffmann RG, Brousseau DC. Low caregiver health literacy is associated with higher pediatric emergency department use and nonurgent visits. Acad Pediatr. 2014;14(3):309-14. 